\title{
Under- and over-investment in education: the role of locked-in fertility
}

\author{
Masao Nakagawa ${ }^{1} \cdot$ Asuka Oura $^{2} \cdot$ Yoshiaki Sugimoto $^{3}$ iD
}

Received: 27 December 2018 / Accepted: 14 January 2021 / Published online: 24 May 2021

(C) The Author(s) 2021

\begin{abstract}
This research argues that, in the presence of idiosyncratic ability shocks after childbirth, irreversible fertility decisions distort the resource allocation between the quantity and quality of children. In underdeveloped environments, where family size is locked into large levels, education investment places a heavy financial burden on households, which deprives some competent children of learning opportunities. In contrast, in more developed environments, family size is locked into smaller levels, which facilitates education investment even for some children with low aptitude. A redistributive policy to mitigate the distortion is proposed for each stage.
\end{abstract}

Keywords Irreversibility · Quantity-quality trade-off · Human capital · Growth

JEL Classification D10 $\cdot$ J13 $\cdot$ J24 $\cdot$ O15

\section{Introduction}

Currently, it is widely recognized that developing skills and knowledge is one of the most effective means not only to improve individual well-being but also to advance the economy as a whole. Indeed, the United Nations has set providing universal access to primary education by 2015 as one of its Millennium Development Goals. Whether developing regions are making full use of this growth strategy is difficult to determine, given that the net enrollment rate in their primary schools was $91 \%$ in

\footnotetext{
Responsible editor: Alessandro Cigno

Yoshiaki Sugimoto

sugimoto@kansai-u.ac.jp

1 Graduate School of Social Sciences, Hiroshima University, Higashihiroshima, Japan

2 Department of Socio-economics, Faculty of Economics, Daito Bunka University, Tokyo, Japan

3 Faculty of Economics, Kansai University, 3-3-35 Yamate-cho, Suita, Osaka, 564-8680, Japan
} 
2015 (United Nations 2016, p. 4) . One of the financial hindrances to the education goal is the increase in the number of potential students, which is expected from the total fertility rate of nearly 3.0 in 2010-2015 in less developed regions, excluding China (United Nations 2017, p. 124). ${ }^{1}$

Regarding advanced countries, it appears that some of them face the opposite situation. In Japan, households cannot afford to maintain replacement-level fertility, partly because of their growing enthusiasm regarding the education of their children. ${ }^{2}$ Despite this situation, $33 \%$ of private universities did not meet their student quota in the 2019 academic year (Promotion and Mutual Aid Corporation for Private Schools of Japan 2019, p. 2). This was partly due to the remarkable supply shift in the previous several decades. ${ }^{3}$ As Clark (2012) indicated, under-enrollment will naturally urge universities to accept a broader range of applicants. This leads to declining quality of higher education. In the USA, education investment yielded negative returns for graduates from $6.5 \%(\approx 120 / 1833)$ of colleges/universities, whereas the total fertility rate dropped to 1.88 during $2010-2015 .{ }^{4}$

A plausible conjecture from these observations is that the resources of those economies are allocated inadequately between the quantity and quality of labor and the bias changes its direction depending on the stage of economic development. In this sense, economies may go through a transition from under- to over-investment in education during the growth process. ${ }^{5}$ The transition is consistent with a recent trend in returns to schooling over the prior decades. Montenegro and Patrinos (2014) estimated returns to schooling from data of 139 economies, revealing a declining trend since the 1980s.

The present paper illuminates the mechanism underlying the aforementioned transition by developing a growth theory that incorporates the irreversibility of fertility decisions. This constraint deteriorates the formation of aggregate human capital, one of the potential forces of economic growth, by restricting the ex post

\footnotetext{
${ }^{1}$ A positive effect of fertility decline on years of schooling was reported by Joshi and Schultz (2013), who assessed a family planning and maternal-child health program in Bangladesh, which was implemented in Matlab. Ashraf et al. (2013) incorporated this effect into a simulation model, and thereby predicted that a lower time path of fertility would lead to a higher path of per capita GDP in Nigeria during the twenty-first century.

${ }^{2}$ According to a questionnaire survey by National Institute of Population and Social Security Research (2017, p. 72), the average planned number of children, 1.87, is lower than the average desired number of children, 2.27, for first-married couples whose wives are between 45 and 49 years of age. For first-married couples of all age groups whose wives are below 50 years, the cost of child rearing and education is the most important reason for this discrepancy (ibid., p. 74).

${ }^{3}$ Indeed, Japanese private universities increased in number to 607 in 2019, a sharp rise from 105 in 1950. See School Basic Survey by the Ministry of Education, Culture, Sports, Science and Technology (MEXT): https://www.mext.go.jp/b_menu/toukei/chousa01/kihon/1267995.htm

${ }^{4}$ See the 2017 college ROI report by PayScale (https://www.payscale.com/college-roi, accessed on October 6, 2017), and also United Nations (2017, p. 132) for the fertility rate.

${ }^{5}$ Under-investment in education refers here to a situation in which aggregate human capital is enhanced by shifting the aggregate resources for child rearing from the quantity to the quality of children. The opposite case applies to over-investment in education. The present paper defines both types of investment from the macroeconomic rather than the individual viewpoint. It is not concerned with skill mismatch between workers and their occupations (see, for example, Sicherman (1991) for this type of mismatch).
} 
quantity-quality trade-off in response to idiosyncratic ability shocks to children. ${ }^{6}$ The present paper also examines how governments could enhance aggregate human capital by redressing the balance of resource allocation.

The long-run, macroeconomic approach of this research distinguishes it from the previous literature. The inequality and growth literature, which has flourished since the 1990s, affirms the possibility of under-investment in human capital in the presence of capital market imperfections (cf. Galor and Zeira 1993, Moav 2002, Mookherjee and Ray 2003). ${ }^{7}$ Other theoretical studies have argued that information imperfections, along with incomplete markets, may induce individuals' precautionary savings for human capital investment (cf. Gould et al. 2001, Aiyagari et al. 2002). ${ }^{8}$ Asymmetric information is another source of under- or over-investment in education. Hopkins (2012) and Cigno and Luporini (2019) demonstrated the theoretical possibility of these phenomena when individuals' abilities are not fully observable to their potential employers. However, none of these findings is fully satisfactory in terms of this paper's objective. The current work uses a macroeconomic framework to account for the emergence of under- and over-investment in education during the growth process, which respectively imply over- and under-investment in fertility.

This research models an overlapping generations economy that features the following key elements. First, individuals derive utility from the quantity and quality of their children as well as from their own consumption, as stated by Becker and Lewis (1973). Second, education investment is more productive for children with higher rather than lower ability. Third, in contrast to the standard literature, there is a time lag between fertility and education decisions, and idiosyncratic ability shocks occur in between. Fertility decisions are assumed to be completely irreversible for ethical, legal, physical, and other reasons. ${ }^{9}$ Once determined, the number of dependents is not adjustable in either direction, and such inflexibility is the source of sunk cost in child rearing. Fourth and finally, consistent with the formulation of Galor and Moav (2000), technological progress is skill-biased in the sense that its acceleration stimulates the incentive for skill acquisition.

\footnotetext{
${ }^{6}$ Goldstein et al. (2003, p. 487, Table 2) compared mean personal ideal family size and mean personal expected family size for young women, using the Eurobarometer 2001 survey. They reported that the former measure is larger than the latter by 0.2 to 0.4 points in major European countries (p. 486). A similar pattern applies to the USA (Hagewen and Morgan 2005, p. 509, Figure 1). These disparities are consistent with this paper's assertion that some households in developed environments are prevented from adjusting their family sizes upward.

${ }^{7}$ Apart from capital market imperfections, Dảvila (2018) argued that the failure to internalize the externality of aggregate human capital fosters social suboptimality of private investment in fertility and in education. In contrast, the present paper attributes inefficiency in the two types of investment to the irreversibility of fertility decisions.

${ }^{8}$ Gould et al. (2001) considered the eroding effect of technological progress, which is biased and random across sectors, on human capital. Aiyagari et al. (2002) highlighted the lack of insurance markets for ability as well as the lack of loan markets.

${ }^{9}$ See, among others, Fraser (2001) and Doepke and Zilibotti (2005) for theoretical arguments underlying the irreversibility of fertility decisions. In relation to schooling, a recent study by (de la Croix and Doepke 2009) focused on the lock-in effect of fertility decisions on individuals' voting preferences to account for the differences in public education systems across countries.
} 
Taking these elements into consideration, the dynamic theory developed later demonstrates a scenario of economic development. In environments characterized by an early developmental stage, where technological progress is sluggish, education investment is not fruitful for parents whose children have average ability. Assuming that children with average ability will be born to them, all households aim to concentrate their child-rearing resources on the quantity of children. ${ }^{10}$ While children reveal their true abilities by the time of schooling, fertility adjustment to a change in education expenses is infeasible at that time. With the locked-in fertility decision, revising the initial education plan involves an unexpected reduction in household consumption.

Accordingly, the irreversibility constraint prevents some households from coping with education costs and the biased allocation of parenting resources entails under-investment in education. To complicate matters, the constraint engenders a counter effect on aggregate human capital: it increases the aggregate amount of parenting resources through the provision of education support, against the initial plan, by households whose children turn out to be significantly competent. The combination of these two opposing forces is generally ambiguous. To mitigate the under-investment in education, the present paper proposes a redistributive policy that subsidizes child education while levying a universal tax on child rearing.

The exogenous acceleration of technological progress eventually alters households' (ex ante) stances toward child rearing. Education investment in this stage is attractive even for parents whose children have average ability. With the aforementioned belief regarding children's abilities, all adult individuals choose smaller family sizes to cope with the cost of future education.

With family sizes locked into small levels, canceling the education plan certainly diminishes the utility from children while it leaves a sufficient budget for consumption. Households therefore invest in education unless their children turn out to be significantly unresponsive to education, leading to over-investment in education. Additionally, those who unexpectedly cancel the education plan shift their budgets away from child rearing. Both of these lock-in effects work adversely on the accumulation of aggregate human capital. In order to mitigate the over-investment, it is useful to stimulate average fertility instead of educating low-ability children, for example, through a universal subsidy for child rearing financed by an ability-based tax on education.

The rest of this paper is organized as follows. Section 2 outlines the structure of the model and then considers optimal decisions on fertility and education, both with and without the irreversibility constraint. These individual child-rearing choices build aggregate human capital as a determinant of final output. Section 3

\footnotetext{
${ }^{10}$ While this strong assumption makes a great contribution to the tractability of the dynamic model, it will not be essential for the outcome of the distorted resource allocations. See Section 4.1 for an extension to the expected-utility framework. Nakagawa and Sugimoto (2011) similarly analyzed the lock-in effect on education decisions by assuming that adult individuals have the same expectations regarding their own abilities.
} 
demonstrates that the constraint fosters either under- or over-investment in education. It also investigates the workings of redistributive policies that are designed to alleviate the biased resource allocation. Section 4 extends the mainline model in Section 2 in some directions and examines how the main results are affected. Section 5 summarizes the analyses and presents some possibilities for future research. The appendix provides mathematical proofs of some key results and supplementary explanations for Section 4.

\section{The model: a locked-in economy}

The economy has an overlapping-generations structure that is closed and abstracted from capital markets. ${ }^{11}$ A single homogeneous good is produced in one sector by employing human capital. Adult individuals have all information except the abilities of the children they intend to have. Ability shocks occur after childbirth, and then parents decide whether to provide education support for their children.

In the "locked-in" economy considered below, family size cannot be either reduced or enlarged once it is decided. ${ }^{12}$ Under these circumstances, there exist households whose reactions to the ability shocks are constrained by their initial plans.

\subsection{Firms}

In perfectly competitive environments, firms generate a single homogeneous good by employing human capital (i.e., efficiency units of labor) with a linear technology. The level of aggregate output, denoted as $Y$, is determined through the production function

$$
Y=A H,
$$

where $A$ and $H$ are the levels of technology and aggregate human capital, respectively. For the sake of simplicity, the price of the final good is normalized to unity. As a result of profit maximization by price-taking firms, $H$ maximizes the aggregate profit $A H-w H$, where $w$ is the market wage rate per unit of human capital. In the competitive labor market considered herein, $w$ is adjusted so that the resulting profit is neither negative nor infinitely large, leading to $w=A$.

\footnotetext{
${ }^{11}$ The baseline model is an extension of the model developed by Galor and Weil (2000), who explored the mechanism underlying the demographic transition in the long-term growth process. In return for allowing the heterogeneity of individuals' abilities, the baseline model needs some modifications in, for example, the household budget constraint and the production function of individual human capital, in order to keep its tractability.

${ }^{12}$ The assumption of perfect irreversibility in the present paper could be relaxed by allowing individuals to have children in two periods, between which unexpected ability shocks occur. The multi-period approach was taken by Iyigun (2000) for different research objectives from the present paper. The author developed a growth model with no uncertainty and demonstrated that the timing of childbearing is delayed by the accumulation of human capital.
} 
Table 1 Definitions of key variables

\begin{tabular}{ll}
\hline Variable & Definition \\
\hline$\delta$ & The fixed cost of raising one child \\
$e^{i}$ & Private spending on education per child \\
$\bar{e}$ & The minimum education cost to acquire advanced skills \\
$g$ & The growth rate of technology \\
$\tilde{a}$ & The critical ability level for education investment \\
$b^{i}$ & The amount of parental resources for child rearing \\
$\delta^{g}$ & The amount of a child-rearing tax \\
& $\left(\delta^{g}>0\right)$ or subsidy $\left(\delta^{g}<0\right)$ \\
$e^{g}$ & $\begin{array}{l}\text { The amount of an education tax } \\
\left(e^{g}>0\right) \text { or subsidy }\left(e^{g}<0\right)\end{array}$ \\
\hline
\end{tabular}

The variables except for $g$ and $\tilde{a}$ are measured in terms of human capital

\subsection{Households}

In the economy there are two generations, adults (parents) and children. They comprise a continuum of individuals existing on the interval $[0, N]$ and on $\left[0, N_{+1}\right]$, respectively.

\subsubsection{Budget constraint and preferences}

Consider the lifetime of individual $i \in[0, N]$. In the first period (childhood), the individual consumes part of parental income to live and possibly to engage in skill acquisition. In the second period (adulthood), the individual acquires $h^{i}>0$ efficiency units of labor to earn wages, while giving birth to $n^{i}$ units of identical children all at once. ${ }^{13}$ Child rearing incurs a cost of $w\left(\delta+e^{i}\right)$ per child, where $\delta>0$ and $e^{i} \geq 0$ are, respectively, the fixed cost and the education cost. ${ }^{14}$ Table 1 summarizes the definitions of key variables used in the present paper.

The remaining income is used up for self-consumption, $c^{i}$, so that no bequests are left to the offspring. It follows that the budget constraint is

$$
c^{i}=w\left[h^{i}-n^{i}\left(\delta+e^{i}\right)\right] .
$$

The utility of adult individual $i, u^{i}$, depends on not only consumption in adulthood but also the aggregate income of his/her children. Each of these children acquires $h_{+1}^{i}$ efficiency units of labor in the subsequent period. With these considerations, the utility function is formulated as

$$
u^{i}=(1-\alpha) \ln c^{i}+\alpha \ln \left(w n^{i} h_{+1}^{i}\right)
$$

where $\alpha \in(0,1)$ measures the degree of parental altruism.

\footnotetext{
${ }^{13}$ Siblings do not have to be born simultaneously. One may assume that when childbirth is sequential, their (identical) ability level is unveiled after the youngest child is born.

${ }^{14}$ Unlike in Galor and Weil (2000) model, the costs of child rearing, $\delta$ and $e^{i}$, are measured not in labor time but in efficiency units of labor, $h^{i}$. The resulting fertility decision depends on $h^{i}$ and, as shown later, aggregate human capital depends on the amount of parenting resources as well as on the efficiency of resource allocation between $n^{i}$ and $e^{i}$. Moav (2005) adopted a hybrid approach by measuring the fixed cost of child rearing in time and the education cost in human capital.
} 


\subsubsection{Production of human capital}

The human capital of a child of parent $i$ depends not only on education $e^{i}$ but also on the child's ability, $a^{i} \in[0,1]$, and on the growth rate of technology between the current and the future period, $g$. It is formed according to the production function

$$
\begin{aligned}
h_{+1}^{i} & =h\left(e^{i}, a^{i}, g\right) \\
& = \begin{cases}\bar{h}-g & \text { if } e^{i}<\bar{e} ; \\
\bar{h}-\left(1-a^{i}\right) g & \text { if } e^{i} \geq \bar{e},\end{cases}
\end{aligned}
$$

where $\bar{h}>0$ is the maximum level of individual human capital and $\bar{e}>0$ is the minimum education cost to acquire advanced skills. It is necessary for $\bar{e}$ to be positive to consider the role of locked-in fertility in education investment. If $\bar{e}$ is substantially small and close to 0 , education cost is modest, regardless of the predetermined family size. In such a case, almost all households invest in education regardless of whether the family size is adjustable or not.

Consistent with the formulation of Galor and Moav (2000), the function $h$ above satisfies three key properties for any $a^{i} \in(0,1)$ and $g>0 .{ }^{15}$ First, education investment has a discrete and positive impact on the formation of human capital. Children become either skilled or unskilled labor depending on the parental education support. ${ }^{16}$ Second, the advantage of skill acquisition is to mitigate the "erosion effect" of technological progress, which makes part of acquired skills obsolete; i.e., $h_{g}\left(0, a^{i}, g\right)<h_{g}\left(\bar{e}, a^{i}, g\right)<0 .{ }^{17}$ These properties engender skill-biased technological progress: an acceleration of technological progress raises the relative skill $h\left(\bar{e}, a^{i}, g\right) / h\left(0, a^{i}, g\right)$, thereby making education investment more advantageous for each child. Third and finally, increased ability enhances human capital if a child receives at least $\bar{e}$ units of education; otherwise, it has no impact on human capital. More precisely, $h_{a}\left(e^{i}, a^{i}, g\right)$ is positive if $e^{i} \geq \bar{e}$ and is 0 if $e^{i}<\bar{e}$. This indicates that, for a given $g>0$, education investment is more advantageous for more competent children.

\subsection{Households' optimization}

In the presence of the unexpected shock, the resource allocation problem of households is divided into two steps. At the time of childbirth (i.e., in ex ante), parents plan for future education investment believing that their newborn children have

\footnotetext{
${ }^{15}$ With respect to the erosion effect below, their theoretical formulation is inspired by Nelson and Phelps (1966).

${ }^{16}$ The discreteness of $h$ with respect to $e^{i}$, which fosters a binary education choice, is not essential for the result of under-investment in education, although it makes the result of over-investment in education less evident. It is the irreversibility of fertility decisions, not the discreteness of $h$, that may limit the ex post adjustment of education. See Section 4.2 for details.

${ }^{17}$ Throughout the present paper, $f_{x}(x, y)$ denotes the partial derivative of a function $f$ with respect to $x$.
} 
average ability. ${ }^{18}$ After childbirth (i.e., in ex post), they unexpectedly find the true ability levels of their children and thus may be inclined to alter their initial plans.

\subsubsection{Unconstrained optimization (benchmark case)}

As a benchmark case, suppose tentatively that at the time of making the education decision, parents can adjust the number of their children as much as they want without any cost. That is, the fertility decision is "unlocked" and reversible. Because this property makes the ex ante optimization meaningless, the model is essentially viewed as a perfect foresight model in which fertility and education decisions are made simultaneously.

Given $h^{i}>0, a^{i} \in[0,1]$ and $g \in[0, \bar{h})$, adult individuals aim to maximize their own utility as price takers. ${ }^{19}$ By substituting Eqs. 2 and 4 into 3, the maximization problem faced by individual $i$ is

$$
\left\{n^{i}, e^{i}\right\}=\arg \max \left\{(1-\alpha) \ln \left[h^{i}-n^{i}\left(\delta+e^{i}\right)\right]+\alpha \ln \left[n^{i} h\left(e^{i}, a^{i}, g\right)\right]\right\},
$$

subject to $\left\{n^{i}, e^{i}\right\} \in \mathbb{R}_{+}^{2}$.

First, consider the fertility decision. The objective function exhibits logarithmic form and strict concavity with respect to $n^{i}$. Hence, the first-order optimality condition yields

$$
n^{i}=\frac{\alpha}{\delta+e^{i}} h^{i},
$$

implying that a fixed fraction of labor, $\alpha$, is devoted to child rearing regardless of the income level (i.e., $\left.n^{i}\left(\delta+e^{i}\right)=\alpha h^{i}\right)$.

Next, consider the education decision. Substitution of Eqs. 6 into 5 reveals that

$$
e^{i}=\arg \max \frac{h\left(e^{i}, a^{i}, g\right)}{\delta+e^{i}},
$$

subject to $e^{i} \geq 0$. As is evident from Eq. 4, the education choice is binary: it is rational for parents to choose either $e^{i}=0$ or $e^{i}=\bar{e}$.

If $g=0, e^{i}=0$ is chosen for any $a^{i} \in[0,1]$ because education investment is not at all productive for anyone; i.e., $h\left(e^{i}, a^{i}, g\right)=\bar{h}$ regardless of education $e^{i}$ and ability $a^{i}$. In contrast, if $g \in(0, \bar{h})$, the education decision is environmentally dependent. To observe this, let $\tilde{a}$ be a critical ability level for which individuals are indifferent between the two options, $e^{i}=0$ and $e^{i}=\bar{e}$. Namely,

$$
\frac{\delta+\bar{e}}{\delta}=\frac{\bar{h}-(1-\tilde{a}) g}{\bar{h}-g},
$$

\footnotetext{
${ }^{18}$ While this strong assumption substantially simplifies the constrained optimization problem, it will not be essential for the aforementioned under-/over-investment in education. See Section 4.1 for an extension to the expected-utility framework.

${ }^{19}$ The condition that $\bar{h}>g$ excludes an unrealistic case in which no education investment leads to a nonpositive amount of human capital.
} 


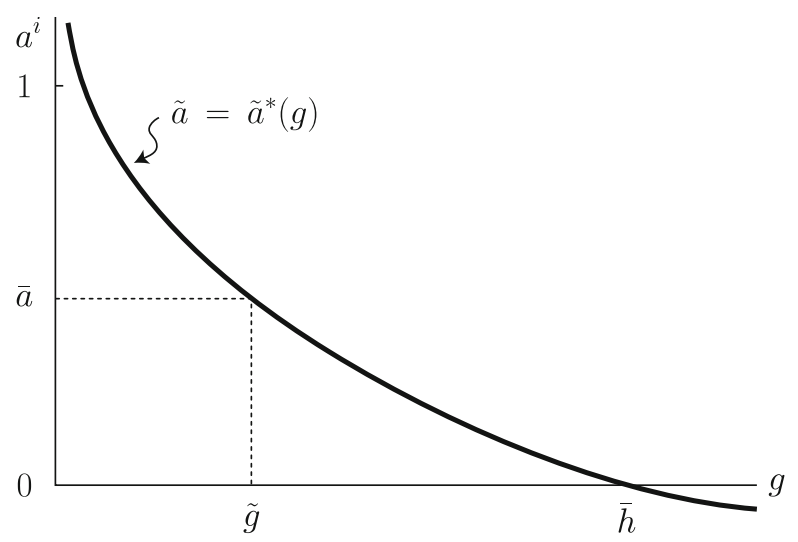

Fig. 1 The critical ability level for education in the unconstrained case. The diagram depicts the negative relationship between the growth rate of technology, $g$, and the critical ability level for education, $\tilde{a}^{*}(g)$. A fall in $\tilde{a}^{*}(g)$ implies a higher ratio of households investing in education in period $t$. The spread of education is attributed to the skill-biased technological progress

using Eqs. 4 and 7. Choosing $\bar{e}$ is strictly preferable if $a^{i}>\tilde{a}$. Solving Eq. 8 for $\tilde{a}$ yields

$$
\tilde{a}=\frac{\bar{e}}{\delta} \cdot \frac{\bar{h}-g}{g} \equiv \tilde{a}^{*}(g),
$$

where $\tilde{a}^{*}(\bar{h})=0$ and $\tilde{a}_{g}^{*}(g)<0 \forall g>0$. This shows that the critical ability $\tilde{a}$ is given by the function $\tilde{a}^{*}(g)$ when the fertility decision is unconstrained. ${ }^{20}$

For analytical simplicity, suppose that individuals do not choose $e^{i}=\bar{e}$ unless it is strictly preferable to $e^{i}=0$. Then, the education decision by individual $i$ is summarized as

$$
e^{i}=e^{*}\left(a^{i}, g\right) \equiv \begin{cases}\bar{e} & \text { if } a^{i}>\tilde{a}^{*}(g) ; \\ 0 & \text { otherwise }\end{cases}
$$

where $g \in[0, \bar{h})$. In contrast to the fertility decision, this decision is independent of his/her human capital $h^{i}$. Figure 1 graphically depicts the function $\tilde{a}^{*}(g)$, which is viewed as the hurdle to education investment for the unconstrained case. A rise in the growth rate of technology $g$ lowers the hurdle because the increased relative skill $h\left(\bar{e}, a^{i}, g\right) / h\left(0, a^{i}, g\right)$ makes education investment more attractive for each parent. Education investment is prevalent to some extent when $\tilde{a}$ is smaller than unity, which is the top ability level in the economy. By contrast, no one invests in education when $\tilde{a} \geq 1$.

\subsubsection{Constrained optimization 1: childbirth and education planning}

Next, consider the ex ante optimization under the irreversibility constraint. Based on the belief that his/her children will have average ability $\bar{a} \in(0,1)$, adult individual $i$

\footnotetext{
${ }^{20}$ In the following, the superscript “* " is attached to functions for the unconstrained case.
} 
decides the quantity of children, $n^{i}$, along with the planned level of education investment, denoted as $e^{p}$. Such an optimization problem can be solved by applying the results for the unconstrained case.

Since $e^{p}$ coincides with the unconstrained education choice for average-ability children, Eq. 10 indicates that

$$
e^{p}=e^{*}(\bar{a}, g)= \begin{cases}\bar{e} & \text { for } g \in(\tilde{g}, \bar{h}) ; \\ 0 & \text { for } g \in[0, \tilde{g}],\end{cases}
$$

where $\tilde{g}$ is, as indicated in Fig. 1 , a critical value such that $\tilde{a}^{*}(\tilde{g})=\bar{a}$. In view of Eq. 9,

$$
\tilde{g}=\frac{\bar{e}}{\bar{a} \delta+\bar{e}} \bar{h}
$$

Then, it follows from Eq. 6 that the fertility decision is

$$
n^{i}=\frac{\alpha}{\delta+e^{p}} h^{i},
$$

where $e^{p}=e^{*}(\bar{a}, g)$. Thus, $e^{p}$ and $n^{i}$ change discontinuously with respect to $g$.

\subsubsection{Constrained optimization 2: education investment}

Next, consider the ex post optimization under the irreversibility constraint. At this time, the individual $i$ has $n^{i}$ units of children whose ability level is $a^{i} \in[0,1]$, and the fertility choice is unchangeable. Applying Eq. 5, one finds that the actual level of education investment is, for given $h^{i}>0, n^{i}>0, a^{i} \in[0,1], g \in[0, \bar{h})$,

$$
e^{i}=\arg \max \left\{(1-\alpha) \ln \left[h^{i}-n^{i}\left(\delta+e^{i}\right)\right]+\alpha \ln h\left(e^{i}, a^{i}, g\right)\right\},
$$

subject to $e^{i} \geq 0$. As is evident from Eq. 4, the education choice is binary and either $e^{i}=0$ or $e^{i}=\bar{e}$ is chosen.

Equation 14 has three notable implications. First, unlike in ex ante optimization, education investment is determined as a trade-off with consumption, $c^{i}$, not with the quantity of children, $n^{i}$. Second, there is no income effect on $e^{i}$. This is because a rise in $h^{i}$ proportionally increases the quantity of children, $n^{i}$, with no impact on the budget constraint in Eq. 2. Third, $n^{i}$ is the source of a sunk cost that makes it difficult to modify the education plan. If $e^{p}=0$ and $\delta$ is sufficiently small, for example, the individual has so many children $\left[n^{i}=\alpha h^{i} / \delta\right.$ from Eq. 13] that he/she cannot afford $e^{i}=\bar{e}$ to them. An extreme case such as this is beyond the scope of the present paper and thus is excluded on the assumption that ${ }^{21}$

$$
\frac{\alpha}{\delta}(\delta+\bar{e})<1
$$

It is obvious from Eq. 4 that if $g=0$, education investment is unproductive and thus $e^{i}=0$ is chosen regardless of the ability level. Now consider the case with

\footnotetext{
${ }^{21}$ Equation A1 is not essential in the sense that its violation would merely exacerbate under-investment in education in the early development stages, with no qualitative influence on over-investment in the later stages (see Lemma 4 below).
} 
$g \in(0, \bar{h})$. Under (A1), there exists a critical ability level, $\tilde{a}^{i}$, for which the individual $i$ is indifferent between the two alternatives, $e^{i}=0$ and $e^{i}=\bar{e}$. In view of Eq. 4, $\tilde{a}^{i}$ is such that

$$
(1-\alpha) \ln \frac{h^{i}-n^{i} \delta}{h^{i}-n^{i}(\delta+\bar{e})}=\alpha \ln \frac{\bar{h}-\left(1-\tilde{a}^{i}\right) g}{\bar{h}-g},
$$

where $n^{i}(\delta+\bar{e})<h^{i}$ from Eqs. 13 and A1. The left side and the right side above represent the utility loss and the utility gain from education investment, respectively, in comparison with the no education case. Regarding the loss, a rise in $n^{i}$ enhances the cost of education, $n^{i} \bar{e}$, and decreases relative consumption. Regarding the gain, a rise in $g$ increases the relative skill and makes education investment more advantageous.

The implicit function above yields

$$
\tilde{a}^{i}=\left\{\left[\frac{h^{i}-n^{i} \delta}{h^{i}-n^{i}(\delta+\bar{e})}\right]^{\frac{1-\alpha}{\alpha}}-1\right\} \frac{\bar{h}-g}{g} \equiv \tilde{a}\left(n^{i}, g\right),
$$

where $\tilde{a}\left(n^{i}, g\right)>0, \tilde{a}_{n}\left(n^{i}, g\right)>0, \tilde{a}_{g}\left(n^{i}, g\right)<0, \tilde{a}(0, g)=0$, and $\tilde{a}\left(n^{i}, \bar{h}\right)=0$ for any $n^{i}<h^{i} /(\delta+\bar{e})$ and $0<g<\bar{h} .^{22}$ One may interpret $\tilde{a}^{i}$ as the hurdle to education investment, in the sense that choosing $\bar{e}$ is strictly preferable if $a^{i}>\tilde{a}^{i}$. The first property, $\tilde{a}\left(n^{i}, g\right)>0$, indicates that the hurdle is not cleared by some households. The function is increasing in $n^{i}$ because a rise in $n^{i}$, as mentioned above, increases the loss from education. Additionally, it is decreasing in $g$ because a rise in $g$ increases the gain from education.

Consequently, the optimal education choice is summarized as

$$
e^{i}=\left\{\begin{array}{l}
\bar{e} \text { if } a^{i}>\tilde{a}\left(n^{i}, g\right) \\
0 \text { otherwise }
\end{array}\right.
$$

where $g \in[0, \bar{h})$. Thus, the ability level $a^{i}$ is the source of heterogeneity in the education decision. Substituting $n^{i}$ from Eqs. 13 into 15 yields

$$
\tilde{a}\left(n^{i}, g\right) \equiv \tilde{a}(g) \equiv \tilde{a} .
$$

This means that all adult individuals have the same criterion for making their education decisions. As shown later, the function $\tilde{a}(g)$ is strictly decreasing and is discontinuous at $\tilde{g}$.

\subsection{Aggregate human capital}

This subsection aggregates human capital of children raised by each household. Focusing on aggregate rather than average human capital allows us to analyze one of the potential engines of economic growth and also to understand the meaning of over-investment in education, accompanied by under-investment in fertility. As will become apparent, the irreversible fertility decision affects the formation of aggregate human capital through households' quantity-quality trade-off.

\footnotetext{
${ }^{22}$ These properties are necessary for Appendix 2. $\tilde{a}\left(n^{i}, g\right)$ is an abbreviation of $\tilde{a}\left(n^{i}, g ; h^{i}\right)$, noting that $\tilde{a}^{i}$ depends on $h^{i}$ as well as on $n^{i}$ and $g$. For simplicity, this notational rule is applied hereafter.
} 
The aggregate amount of human capital in the subsequent period is expressed as

$$
H_{+1}=\int_{0}^{N} n^{i} h_{+1}^{i} d i .
$$

As Lemma 1 below confirms, the unconstrained parental decision maximizes the aggregate human capital of each household, $n^{i} h_{+1}^{i}$, and thus that of the economy, $H_{+1}$.

Lemma 1 In the absence of the irreversibility constraint, consider individual $i$ who aims to allocate $b^{i}$ efficiency units of labor for child rearing; i.e., $b^{i} \equiv\left(\delta+e^{i}\right) n^{i}$. Given $g>0$ and $b^{i}>0 \forall i \in[0, N]$, the education choice $e^{i}=e^{*}\left(a^{i}, g\right)$ maximizes aggregate human capital in the subsequent period.

Proof Note that $n^{i}=b^{i} /\left(\delta+e^{i}\right)$ from the resource constraint. Substituting this result and Eqs. 4 into 18 yields

$$
H_{+1}=\int_{0}^{N} b^{i} \frac{h\left(e^{i}, a^{i}, g\right)}{\delta+e^{i}} d i,
$$

where $b^{i}$ is exogenous. Since the education decision $e^{i}=e^{*}\left(a^{i}, g\right)$ satisfies Eq. 7, it maximizes the integral in Eq. 19 and thus $H_{+1}$.

Equation 19 shows that aggregate human capital $H_{+1}$ depends on two factors: the amount of parenting resources, $b^{i}$, and the efficiency of its allocation between children's quantity and quality. As will become apparent, these are the channels through which the irreversibility of fertility decision-making affects human capital accumulation.

It is shown below that the irreversibility constraint makes room for increasing aggregate human capital by reallocating child-rearing resources between the quantity and the quality of children. Under-investment in education, or equivalently overinvestment in fertility, occurs if $e^{i} \leq e^{*}\left(a^{i}, g\right) \forall i \in[0, N]$ with strict inequality for some $i$. This is the situation in which aggregate human capital $H_{+1}$ is enhanced by allocating someone's $b^{i}$ more to $e^{i}$ (and less to $n^{i}$ ). Conversely, over-investment in education, or equivalently under-investment in fertility, occurs if $e^{i} \geq e^{*}\left(a^{i}, g\right)$ $\forall i \in[0, N]$ with strict inequality for some $i$.

\section{Analysis}

This section demonstrates two stages of economic development, referred to as stages I and II, created by exogenous technology growth. The irreversibility constraint affects the amount of resources available to children and its allocation between their quantity and quality. Such lock-in effects change qualitatively depending on the development stage.

The focus here is not on underdeveloped environments, in which some households rely on child labor. The analysis below is limited to the situation in which at least 
some households invest in child education; i.e., $\tilde{a}(g)<1 .^{23}$ Such a case occurs when $g$ is greater than a critical level $\hat{g}$, which is defined later in Eq. 22. This critical value, along with $\tilde{g}$ in Eq. 12, distinguishes the aforementioned two stages.

\subsection{Stage I: under-investment in education}

Stage I, where $\hat{g}<g \leq \tilde{g}$, involves under-investment in education, i.e., a biased allocation of parenting resources toward the quantity of children. Each household make a fertility decision with no prospect of future education investment. The resulting family size, locked into a large level, scales up the potential cost of schooling. Under these circumstances, households adhere to the initial plan unless their children turn out to be significantly competent.

\subsubsection{Households' decisions on child rearing}

According to Eqs. 11 and 13, the education plan and the fertility choice of individual $i$ are, for $g \leq \tilde{g}$,

$$
e^{p}=0 ; \quad n^{i}=\alpha h^{i} / \delta .
$$

Thus, in stage I, households have no education plan and invest all child-rearing resources in the quantity of children. Given the fertility decision, Eq. 17 reveals that the critical ability level for the ex post education decision is

$$
\tilde{a}=\kappa^{I} \cdot \frac{\bar{h}-g}{g} \equiv \tilde{a}^{I}(g),
$$

where

$$
\kappa^{I} \equiv\left[\frac{(1-\alpha) \delta}{(1-\alpha) \delta-\alpha \bar{e}}\right]^{\frac{1-\alpha}{\alpha}}-1
$$

Since $\kappa^{I}>0$ under the condition in Eq. A1, it follows that $\tilde{a}^{I}(\hat{g})=1, \tilde{a}^{I}(\bar{h})=0$, and $\tilde{a}_{g}^{I}(g)<0 \forall g>0$, where

$$
\hat{g} \equiv \kappa^{I} \bar{h} /\left(1+\kappa^{I}\right) .
$$

By definition, $\hat{g}$ is the lower limit of stage I and, in contrast to $\tilde{g}$ in Eq. 12, it is the critical value for the ex post decision.

Stage I, where $\hat{g}<g \leq \tilde{g}$, is the situation in which at least some households overturn the initial education plan in Eq. 20. That is, the irreversibility constraint is not too strong to prevent their ex post adjustments. The existence of the stage builds on the assumption that

$$
\hat{g}<\tilde{g}
$$

\footnotetext{
${ }^{23}$ This paper's objective is to investigate the role of the irreversibility constraint in the education decision and economic growth. The constraint would be unbinding in underdeveloped environments in which households do not invest in education regardless of the constraint; i.e., $1<\tilde{a}^{*}(g)<\tilde{a}(g)$ in Fig. 2. Although the constraint is binding for some households when $\tilde{a}^{*}(g)<1<\tilde{a}(g)$, the present paper does not address this case for analytical simplicity.
} 


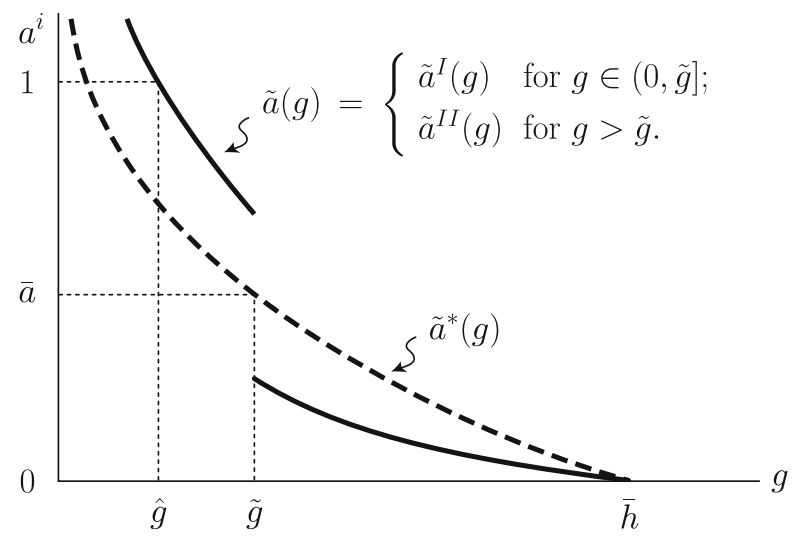

Fig. 2 The critical ability level for education in the constrained case. The diagram depicts the relationship between the growth rate of technology and the critical ability level for education in the locked-in economy, $\tilde{a}(g)$, in comparison with the one for the unconstrained case, $a^{*}(g)$. While both of the functions decrease as $g$ increases, their quantitative relationship reverses when $g$ crosses over $\tilde{g}$. The function $\tilde{a}^{I I}(g)$ is specified later in Eq. 28

where the inequality holds if the education cost $\bar{e}$ is sufficiently small. ${ }^{24}$

Lemma $2 \operatorname{Under}(\mathrm{A} 1), \tilde{a}^{I}(g)>\tilde{a}^{*}(g)>0 \forall g \in(0, \bar{h})$.

Proof See Appendix 1.

Lemma 2 asserts that the hurdle to education in stage I is placed above the benchmark level $\tilde{a}^{*}(g)$. This result holds because with the fertility choice in stage I, locked into a high level, the potential cost of education $\bar{e} n^{i}$ places a heavy financial burden on households.

Figure 2 reflects the properties of $\tilde{a}^{I}(g)$ shown so far. The negative slope of $\tilde{a}^{I}(g)$ is due to the skill-biased technological progress. A rise in $g$ works in favor of skilled workers and thus makes education investment more attractive for each household.

\subsubsection{The lock-in effect on aggregate human capital}

Figure 3 illustrates the lock-in effects on ex post decisions of each adult individual in stage I. The solid line and the broken line indicate the cases with and without the irreversibility constraint, respectively. Noting that $e^{i} \leq e^{*}\left(a^{i}, g\right)$ for all $a^{i} \in$ $[0,1]$ with strict inequality for $a^{i} \in\left(\tilde{a}^{*}(g), \tilde{a}^{I}(g)\right]$, one finds that the constraint prevents education investment by some households.

\footnotetext{
${ }^{24}$ Recalling that $\hat{g} \equiv \kappa^{I} \bar{h} /\left(1+\kappa^{I}\right)$ and $\tilde{g} \equiv \bar{e} \bar{h} /(\bar{a} \delta+\bar{e})$, one may rewrite the condition $\hat{g}<\tilde{g}$ as $\bar{a}<\bar{e} /\left(\delta \kappa^{I}\right)$, where $0<\bar{a}<1$ by assumption and $\bar{e} /\left(\delta \kappa^{I}\right)<1$ from Lemma 2 immediately below. This condition is satisfied if $\bar{e}>0$ is sufficiently small because, given L'Hôpital's rule, $\bar{e} /\left(\delta \kappa^{I}\right)$ approaches unity as $\bar{e}$ approaches 0 . Choosing such a small $\bar{e}$ is consistent with Eq. A1.
} 


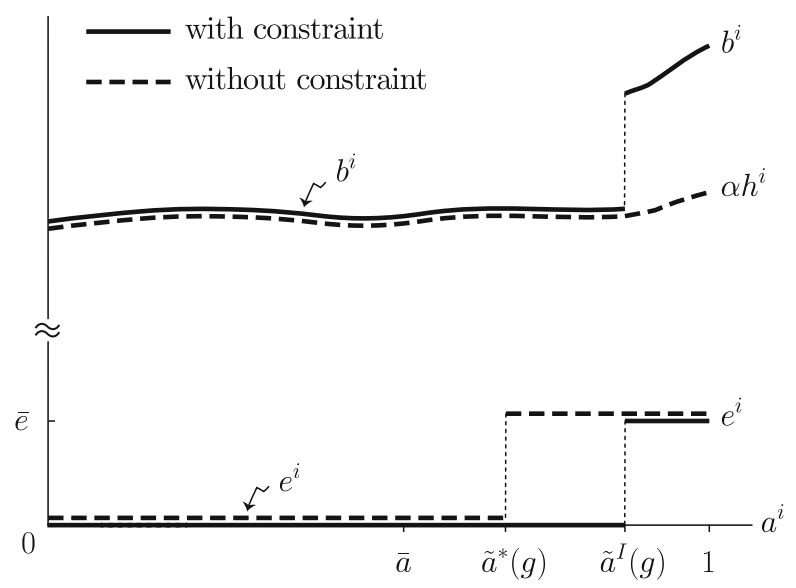

Fig. 3 The lock-in effects on education and parenting resources in stage I. The diagram depicts the lockin effects on the amount of efficient labor devoted to children, $b^{i}$, and on the education decision, $e^{i}$. The irreversibility constraint induces households in the interval $\left(\tilde{a}^{I}(g), 1\right]$ to spend more on their children, whereas it prevents those in $\left(\tilde{a}^{*}(g), \tilde{a}^{I}(g)\right]$ from investing in child education

If fertility decisions were reversible, households on $\left(\tilde{a}^{*}(g), \tilde{a}^{I}(g)\right]$ would reduce their family sizes to finance the cost of schooling. ${ }^{25}$ However, such an adjustment is in fact infeasible and education investment would incur a fall in consumption. The ability shocks to those households are not large enough for them to make such sacrifices.

As Lemma 1 claims, the unconstrained decision is optimal for the formation of aggregate human capital, $H_{+1}$ in Eq. 19, regardless of the child-rearing budget $b^{i}$. Therefore, according to the definition following Lemma 1, Fig. 3 shows the economy facing under-investment in education or, equivalently, over-investment in fertility.

Despite the inefficient resource allocation, the irreversibility constraint has an ambiguous effect on $H_{+1}$ because it also increases child-rearing expenses for some households. This counterforce is represented by Fig. 3. From one perspective, households that receive a shock $a^{i} \in\left[0, \tilde{a}^{I}(g)\right]$ follow the quantity-oriented plan in Eq. 20 and thus spend a fixed fraction of income on child rearing; i.e., $b^{i}=$ $(\delta+0) \alpha h^{i} / \delta=\alpha h^{i}$, as in the unconstrained case in Eq. 6. From another perspective, those with $a^{i} \in\left(\tilde{a}^{I}(g), 1\right]$ invest in education as opposed to the initial plan. Because their family sizes cannot be reduced accordingly, the upward revision of the education plan requires more than $\alpha h^{i}$ efficiency units of labor for their children; i.e., $b^{i}=(\delta+\bar{e}) \alpha h^{i} / \delta>\alpha h^{i}$. Such an adjustment at the expense of consumption is made only if the observed ability level is sufficiently high.

\footnotetext{
${ }^{25}$ The discontinuity of $h\left(e^{i}, a^{i}, g\right)$ with respect to $e^{i}$ is not essential for the lock-in effect on education decisions. If the function $h$ was alternatively continuous with respect to $e^{i}$, the reaction function $e\left(a^{i}, g\right)$ would also be continuous with respect to $a^{i}$. Then, the irreversibility constraint would make the education reaction less sensitive to ability shocks. See Section 4.2 for details.
} 
To summarize, the increase in child-rearing expenses has a positive impact on aggregate human capital, thereby negating the adverse effect of under-investment in education. The result establishes the following proposition.

Proposition 1 (The lock-in effect in stage I) Under (A1), the irreversibility constraint on fertility decisions has an ambiguous effect on aggregate human capital $H_{+1}$ in stage $I$.

\subsubsection{A redistribution policy}

This subsection examines the possibility of a redistribution policy that reallocates parenting resources between the quantity and quality of children and thereby enhances aggregate human capital in stage I.

Suppose that the government imposes a tax $w \delta^{g}$ on raising one child and provides a subsidy $-w e^{g}$ for sending one child to school, where $\delta^{g} \geq 0$ and $e^{g} \leq 0 .{ }^{26}$ That is, the taxation is an obligation for all households (and thus is viewed as a poll tax), whereas the subsidy is targeted only at investors in child education. It follows from Eq. 15 that the critical ability level that leaves parent $i$ indifferent between the two education choices, 0 and $\bar{e}$, is

$$
\tilde{a}^{i}=\left\{\left[\frac{h^{i}-n^{i}\left(\delta+\delta^{g}\right)}{h^{i}-n^{i}\left(\delta+\delta^{g}+\bar{e}+e^{g}\right)}\right]^{\frac{1-\alpha}{\alpha}}-1\right\} \frac{\bar{h}-g}{g} .
$$

The policy scheme is announced before individuals give birth to children, and $\delta^{g}$ is small enough to keep the economy in stage I. Equation 20 is then modified to

$$
e^{p}=0 ; \quad n^{i}=\frac{\alpha}{\delta+\delta^{g}} h^{i} .
$$

As expected intuitively, a rise in $\delta^{g}$ increases the fixed cost of child rearing, thereby locking $n^{i}$ into a lower level. Substituting the results of Eqs. 24 into 23 yields

$$
\tilde{a}^{i}=\left\{\left[\frac{(1-\alpha)\left(\delta+\delta^{g}\right)}{(1-\alpha)\left(\delta+\delta^{g}\right)-\alpha\left(\bar{e}+e^{g}\right)}\right]^{\frac{1-\alpha}{\alpha}}-1\right\} \frac{\bar{h}-g}{g} \equiv \tilde{a},
$$

implying that $\tilde{a}^{i}$ is shared by all households.

The government has full knowledge of the entire economy while it cannot identify the abilities of unborn children. $a^{i}$ is identically and independently distributed across households and generations according to a cumulative distribution function $F{ }^{27}$ As a result of the government's balanced budget,

$$
e^{g}=\frac{-\delta^{g}}{1-F(\tilde{a})} \leq 0,
$$

\footnotetext{
${ }^{26}$ As shown by Table $1, \delta^{g}$ plays a role of taxation when $\delta^{g}>0$ and $e^{g}$ works as an education subsidy when $e^{g}<0$.

${ }^{27}$ The function has standard properties such that $F(a)=0 \forall a \leq 0, F(a)=1 \forall a \geq 1$, and $F^{\prime}(a)>0$ $\forall a \in(0,1)$.
} 
where $1-F(\tilde{a})$ is the fraction of subsidy recipients among adult individuals. ${ }^{28}$ It then follows that $\delta^{g}+e^{g} \leq 0$ in Eq. 23; namely, the redistribution policy reduces the net cost of raising a child as skilled labor. In the case of no taxation, $\delta^{g}=e^{g}=0$ and thus $\tilde{a}$ in Eq. 25 is given by $\tilde{a}^{I}(g)$ in Eq. 21 .

Therefore, in the vicinity of any $g>0$ and $\delta^{g}=0$, the critical ability level $\tilde{a}$ in Eq. 25 is expressed as a single-valued function

$$
\tilde{a}=\tilde{a}^{I}\left(g ; \delta^{g}\right),
$$

where $\tilde{a}^{I}(g ; 0)=\tilde{a}^{I}(g)$.

Now consider a redistribution policy that increases $\delta^{g}$ marginally at $\delta^{g}=0$. The policy effect on $\tilde{a}$ is given by Lemma 3 below, in which $\partial \tilde{a}^{I}(g ; 0) / \partial \delta^{g}$ denotes the derivative $\partial \tilde{a}^{I}\left(g ; \delta^{g}\right) / \partial \delta^{g}$ evaluated at $\delta^{g}=0 .{ }^{29}$

Lemma 3 Under (A1), $\partial \tilde{a}^{I}(g ; 0) / \partial \delta^{g}<0 \forall g \in(\hat{g}, \bar{h})$.

\section{Proof See Appendix 1.}

The lemma shows that the redistribution policy pushes down the hurdle to education, $\tilde{a}$. The policy effect is explained by two factors in Eq. 23: the quantity of children, $n^{i}$, and the unit cost of child rearing, $\delta+e^{i}$. First, given Eq. 24, the change in $\delta^{g}$ increases the fixed cost of child rearing, thereby locking the quantity of children $n^{i}$ into a lower level. Second, given Eq. 26, the increase in $\delta^{g}$ makes raising a child as unskilled labor more expensive and raising a child as skilled labor less expensive. The policy accordingly narrows the interval $\left(\tilde{a}^{*}(g), \tilde{a}^{I}(g)\right]$ in Fig. 3 and mitigates under-investment in education. ${ }^{30}$

\subsection{Stage II: over-investment in education}

Stage II, where $g>\tilde{g}$, is characterized by over-investment in education, i.e., a biased allocation of parenting resources toward the quality of children. As a result of the fertility decision with a view to education spending, the family size is locked into a smaller level. Under these circumstances, households invest in education as planned unless their children turn out to be significantly unsuited to education.

\footnotetext{
${ }^{28}$ With a balanced budget, the redistribution policy has no direct influence on the total amount of households' budgets for child rearing, $B$, because$$
B \equiv \int_{0}^{N} b^{i} d i=\left\{\delta+\delta^{g}+[1-F(\tilde{a})]\left(\bar{e}+e^{g}\right)\right\} \int_{0}^{N} n^{i} d i .
$$

${ }^{29}$ This notation applies to other functions hereafter.

${ }^{30}$ Since $\tilde{a}^{*}(g)$ in Lemma 2 and Fig. 3 is the critical ability level for the education decision in the benchmark case, it is immune from any policies executed for the locked-in economy considered here. This is also the case for Lemma 4 and Fig. 4 below. In contrast, $\tilde{a}^{*}(g)$ used to define $\tilde{g}$ in Eq. 12 is the critical ability level in ex ante optimization, rather than in the benchmark case, and is thus influenced by those policies.
} 


\subsubsection{Households' decisions on child rearing}

Given Eqs. 11 and 13, the education plan and the fertility choice of individual $i$ is

$$
e^{p}=\bar{e} ; \quad n^{i}=\frac{\alpha h^{i}}{\delta+\bar{e}} .
$$

Thus, in stage II, all households plan to invest in education by choosing smaller family sizes for a given amount of income. Then, Eq. 17 shows that the critical ability level for the ex post education decision is

$$
\tilde{a}=\kappa^{I I} \cdot \frac{\bar{h}-g}{g} \equiv \tilde{a}^{I I}(g)
$$

where

$$
\kappa^{I I} \equiv\left[\frac{(1-\alpha) \delta+\bar{e}}{(1-\alpha)(\delta+\bar{e})}\right]^{\frac{1-\alpha}{\alpha}}-1>0 .
$$

Figure 2 depicts the properties of $\tilde{a}^{I I}(g)$ considering the result of Lemma 4 below.

Lemma $40<\tilde{a}^{I I}(g)<\tilde{a}^{*}(g) \forall g \in(0, \bar{h})$.

Proof See Appendix 1.

Lemma 4 indicates that the hurdle to education in stage II is lower than the benchmark level. This result is intuitive because the fertility choice in stage II, which is locked into a low level, curtails the potential cost of education for each household, $\bar{e} n^{i}$.

\subsubsection{The lock-in effect on aggregate human capital}

Figure 4 graphically represents the lock-in effect on the ex post parental choices in stage II. There is a partial gap between the solid line and the broken line, which respectively indicate the optimal choices with and without the irreversibility constraint. Since $e^{i} \geq e^{*}\left(a^{i}, g\right)$ for all $a^{i} \in[0,1]$ with strict inequality for $a^{i} \in\left(\tilde{a}^{I I}(g), \tilde{a}^{*}(g)\right]$, the constraint induces some households to invest in education. Recalling Lemma 1 again, one finds that the economy in stage II suffers from over -investment in education or, equivalently, under-investment in fertility. ${ }^{31}$

While households on $\left(\tilde{a}^{I I}(g), \tilde{a}^{*}(g)\right]$ spend on education as planned in Eq. 27, they would not carry out the plan if the family size could be enlarged after receiving the negative ability shocks. Since such an ex post adjustment is in fact infeasible, the fertility decision in this stage lightens the financial burden of education, $\bar{e} n^{i}$. Under

\footnotetext{
${ }^{31}$ In this situation, education investment is not sufficiently productive for some workers. Since those workers earn wages appropriate to their skill levels, they are categorized as "apparently over-educated workers" in Chevalier (2003). The author defines apparently over-educated workers as graduates who are satisfied with a non-graduate job and genuinely over-educated workers as those who are not. Using data on UK graduates from 1985 and from 1990, he reported that 483 out of 4844 graduates fell into the former category (p. 514, Table 1).
} 


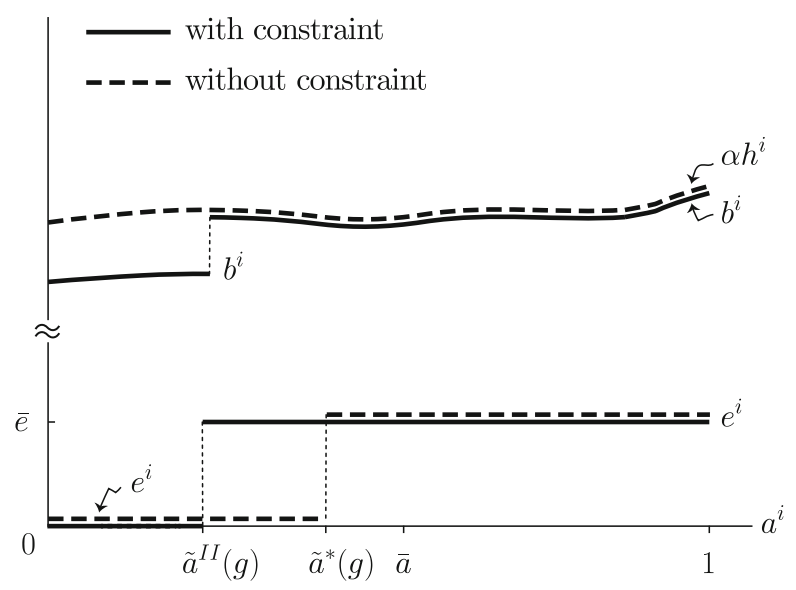

Fig. 4 The lock-in effects on education and parenting resources in stage II. In contrast to Fig. 3, the diagram shows that the irreversibility constraint induces households in the interval $\left[0, \tilde{a}^{I I}(g)\right]$ to spend less on their children, whereas it induces those in $\left(\tilde{a}^{I I}(g), \tilde{a}^{*}(g)\right]$ to invest in education

these circumstances, the ability shocks they receive are not large enough for them to cancel the initial plan.

There is another channel through which the irreversibility constraint adversely affects aggregate human capital $H_{+1}$ in Eq. 19. Unlike in the previous stage, it decreases the child-rearing budget $b^{i}$ for some households. This effect is illustrated by Fig. 4. Households that observe $a^{i} \in\left(\tilde{a}^{I I}(g), 1\right]$ follow the initial plan and devote $\alpha h^{i}$ efficiency units of labor to child rearing as in the benchmark case. In contrast, those with $a^{i} \in\left[0, \tilde{a}^{I I}(g)\right]$ find that observed abilities are too low to carry out the education plan in Eq. 27. Because the number of their children cannot be increased correspondingly, their ex post decision leads to a smaller budget for children than initially planned; i.e., $b^{i}=(\delta+0) \alpha h^{i} /(\delta+\bar{e})<\alpha h^{i}$.

All things considered, the lock-in effect on human capital accumulation is necessarily negative in stage II. The following proposition is now derived.

Proposition 2 Under (A1), the irreversibility constraint on fertility decisions reduces aggregate human capital $H_{+1}$ in Stage II.

\subsubsection{A redistribution policy}

This subsection designs another type of redistribution policy that improves overinvestment in education and stimulates fertility. In this case, the government provides a subsidy $-w \delta^{g}$ for raising one child as parenting support while imposing a tax $w e^{g}$ on sending a low-ability child to higher education, where $\delta^{g} \leq 0$ and $e^{g} \geq 0 .{ }^{32}$ One may interpret the education tax considered here as the ability-based provision of a

\footnotetext{
${ }^{32}$ As before, the policy is not so drastic that the economy leaves stage II.
} 
public scholarship. In order to encourage child bearing, suppose that the education tax is targeted at households whose children are below average in ability. ${ }^{33}$

When the policy scheme is initially announced, none of the adult individuals expects to be subject to taxation. The ex ante decisions given by Eq. 27 are therefore modified to

$$
e^{p}=\bar{e} ; \quad n^{i}=\frac{\alpha}{\delta+\delta^{g}+\bar{e}} h^{i} .
$$

Decreasing $\delta^{g}$ lightens the fixed cost of child rearing, thereby locking $n^{i}$ into a higher level.

Turning to the ex post education decision, recall that households that are indifferent in their education decisions necessarily have below-average children; i.e., $\tilde{a}=\tilde{a}^{I I}(g)<\bar{a}$ in Fig. 4. Because the households would be subject to taxation if they invested in education, one may reuse Eqs. 23 with 29 to obtain

$$
\tilde{a}^{i}=\left\{\left[\frac{(1-\alpha)\left(\delta+\delta^{g}\right)+\bar{e}}{(1-\alpha)\left(\delta+\delta^{g}+\bar{e}\right)-\alpha e^{g}}\right]^{\frac{1-\alpha}{\alpha}}-1\right\} \frac{\bar{h}-g}{g} \equiv \tilde{a} .
$$

Under these circumstances, the balanced budget constraint faced by the government yields

$$
e^{g}=\frac{-\delta^{g}}{F(\bar{a})-F(\tilde{a})} \geq 0,
$$

where the denominator is the fraction of households paying the education tax. The ability distribution is fully known to the government. Increasing the subsidy $-\delta^{g}$ requires a heavier taxation burden, and the overall effect is $\delta^{g}+e^{g} \geq 0$; namely, the redistribution policy increases the net cost of raising a child with below average ability as skilled labor. In addition, it is clear that if $\delta^{g}=0, e^{g}=0$ and thus $\tilde{a}$ in Eq. 30 is given by $\tilde{a}^{I I}(g)$ in Eq. 28 .

Thus, in the vicinity of any $g>0$ and $\delta^{g}=0$, the critical ability level $\tilde{a}$ in Eq. 30 is expressed as a single-valued function such that

$$
\tilde{a}=\tilde{a}^{I I}\left(g ; \delta^{g}\right),
$$

where $\tilde{a}^{I I}(g ; 0)=\tilde{a}^{I I}(g)$. The function has the following property.

Lemma $5 \partial \tilde{a}^{I I}(g ; 0) / \partial \delta^{g}<0 \forall g \in(\tilde{g}, \bar{h})$.

\section{Proof See Appendix 1.}

Lemma 5 shows that a redistribution policy that decreases $\delta^{g}$ marginally at $\delta^{g}=$ 0 lifts the hurdle to education investment, $\tilde{a}$. The policy effect is explained by two factors in Eq. 23: the quantity of children, $n^{i}$, and the unit cost of child rearing, $\delta+e^{i}$. First, given Eq. 29, the change in $\delta^{g}$ reduces the fixed cost of child rearing,

\footnotetext{
${ }^{33}$ More generally, one may choose any ability level on $[\tilde{a}, \bar{a}]$ as the critical level for the education tax. Alternatively, if an ability level on $(\bar{a}, 1]$ is chosen as the critical level, all adult individuals in stage II expect to be subject to taxation at the time of childbirth. Such a policy would reduce rather than increase the quantity of children they intend to raise. For this reason, this section does not address an unconditional education policy as in stage I.
} 
thereby locking the quantity of children $n^{i}$ into a higher level. Second, given Eq. 31, the decrease in $\delta^{g}$ makes raising a child as unskilled labor less expensive and a low ability child as skilled labor more expensive. The policy accordingly narrows the interval $\left(\tilde{a}^{I I}(g), \tilde{a}^{*}(g)\right]$ in Fig. 4 and mitigates over-investment in education.

\section{Some extensions}

This section extends the mainline model developed in Section 2 by relaxing three key assumptions. It is shown that the central result of the present paper-the emergence of under- and over-investment in education-is robust against the extensions as long as fertility decisions are irreversible.

\subsection{Expected utility}

The first extension is to take the standard expected utility approach. Instead of the belief that children are born with average ability, suppose that individuals aim to maximize their utility expected from the ability distribution that is, for simplicity, uniform over $[0,1]$. The uncertainty disappears between childbirth and education decision.

The optimization problem under these circumstances can be solved backwards. Because there is no change in the ex post education decision in Eq. 16, the analysis here focuses on the preceding fertility decision as the potential influence on the economy.

\subsubsection{Constrained optimization: childbirth}

Consider the optimal fertility choice of adult individual $i$ before $a^{i}$ is unveiled. $n^{i}$ is chosen to maximize his/her expected utility by taking into account the ex post decision in Eq. 16 along with Eqs. 2-4. It follows that

$$
\begin{aligned}
n^{i}= & \arg \max \left\{\alpha \ln n^{i}+\int_{0}^{\tilde{a}^{i}}\left[(1-\alpha) \ln \left(h^{i}-n^{i} \delta\right)+\alpha \ln (\bar{h}-g)\right] d a\right. \\
& \left.+\int_{\tilde{a}^{i}}^{1}\left[(1-\alpha) \ln \left(h^{i}-n^{i}(\delta+\bar{e})\right)+\alpha \ln (\bar{h}-(1-a) g)\right] d a\right\},
\end{aligned}
$$

where $\tilde{a}^{i}=\tilde{a}\left(n^{i}, g\right)$ and $0<g<\bar{h}$. The optimization problem is divided into several cases depending on whether $\tilde{a}^{i}$ is greater than unity. Appendix 2 shows that the optimal fertility choice is, in contrast to Eq. 13, expressed as a continuous function of $g$ kinked at $\hat{g}$. More precisely,

$$
n^{i}=\gamma(g) h^{i}
$$

where $\gamma(g)=\alpha / \delta \forall g \in(0, \hat{g}], \gamma^{\prime}(g)<0 \forall g \in(\hat{g}, \bar{h})$, and $\gamma(\bar{h})=\alpha /(\delta+\bar{e})$. This shows that $n^{i}$ may fall between the two optimal levels in the mainline model. The possibility of an interior solution is because the objective function in Eq. 32 takes into account all ability levels and thus two education choices, $e^{i}=0$ and $e^{i}=\bar{e}$, in contrast to the previous approach employed in Section 2.3.2. 


\subsubsection{The critical ability for education}

Given Eqs. 33, 15 reveals that

$$
\tilde{a}^{i}=\kappa(g) \frac{\bar{h}-g}{g} \equiv \tilde{a}(g),
$$

where

$$
\kappa(g) \equiv\left[\frac{1-\gamma(g) \delta}{1-\gamma(g)(\delta+\bar{e})}\right]^{\frac{1-\alpha}{\alpha}}-1 .
$$

In view of Eqs. 21 and 28, $\kappa(g)$ is a continuous function such that $\kappa(g)=\kappa^{I} \forall g \in$ $(0, \hat{g}], \kappa^{\prime}(g)<0 \forall g \in(\hat{g}, \bar{h})$, and $\kappa(\bar{h})=\kappa^{I I}$. Thus, unlike in Fig. $2, \tilde{a}(g)$ in Eq. 34 is continuous at any point on $(0, \bar{h})$, while it has a negative slope and $\tilde{a}(\hat{g})=1$ as before.

Correspondingly, let us redefine $\tilde{g}$ as a critical value of $g$ for which $\tilde{a}(g)=\tilde{a}^{*}(g)$ or equivalently $\kappa(g)=\bar{e} / \delta$, noting Eqs. 9 and 34. Since $\kappa^{I I}<\bar{e} / \delta<\kappa^{I}$ as implied by Lemmas 2 and 4 , the properties of $\kappa(g)$ ensure that $\tilde{g}$ exists uniquely and

$$
\tilde{a}(g) \begin{cases}>\tilde{a}^{*}(g) & \text { for } g \in(0, \tilde{g}) ; \\ =\tilde{a}^{*}(g) & \text { for } g=\tilde{g} ; \\ <\tilde{a}^{*}(g) & \text { for } g \in(\tilde{g}, \bar{h}) .\end{cases}
$$

Since this is a counterpart to Lemmas 2 and 4 , one finds that either under- or overinvestment in education occurs depending on whether $g$ is greater than $\tilde{g}$. Therefore, the expected-utility optimization does not alter the main result qualitatively even though it affects the education decision through the moderate fertility choice. The key factor here is the irreversibility constraint rather than the process of making the fertility choice. ${ }^{34}$

\subsection{Continuity of education}

This subsection examines the case in which households can spend any positive amount of resources on education investment. One may wonder if such continuity of education, which appears to be persuasive particularly at non-compulsory levels, nullifies the irreversibility constraint.

Suppose that the production function of human capital, $h\left(e^{i}, a^{i}, g\right)$, is now continuous with respect to $e^{i}$. Technically speaking, for $e^{i} \in(0, \infty), a^{i} \in(0,1)$ and $g \in(0, \infty)$,

$$
\begin{gathered}
h_{e}>0 ; \quad h_{g}<0 ; \quad h_{e e}<0 ; \quad h_{e g}>0 ; \\
G_{a}^{*}\left(e^{i}, a^{i}, g\right)>0 ; \\
G^{*}\left(0, a^{i}, 0\right)<0<\lim _{g \rightarrow \infty} G^{*}\left(0, a^{i}, g\right),
\end{gathered}
$$

\footnotetext{
${ }^{34}$ The irreversibility of fertility decision also plays an important role in the theoretical literature on mortality shocks and a precautionary demand for the number of children (cf. Kalemli-Ozcan (2003) and (Doepke 2005)). While the mortality rate in their papers is exogenous, this subsection allows individuals to control the probability of investing in education.
} 
where

$$
G^{*}\left(e^{i}, a^{i}, g\right) \equiv\left(\delta+e^{i}\right) h_{e}\left(e^{i}, a^{i}, g\right)-h\left(e^{i}, a^{i}, g\right) .
$$

The last condition is necessary to divide the development process into two stages as in Section 3. With no other structural changes from the mainline model, this extension has no influence on the fertility decisions in Eqs. 6 and 13.

Consider the case of unconstrained optimization. Given Eq. 7, the first-order condition for $e^{i}$ is $G^{*}\left(e^{i}, a^{i}, g\right) \leq 0$, with equality if $e^{i}>0$. Now redefine $\tilde{a}$ as a critical ability level for which $G^{*}\left(0, a^{i}, g\right)=0$, so that $\tilde{a}$ is given by a decreasing function $\tilde{a}^{*}(g) .{ }^{35}$ Using this function, one may write the education decision as a continuous function $e^{*}\left(a^{i}, g\right)$ such that, for $a^{i} \in[0,1]$ and $g \in[0, \infty)$,

$$
e^{*}\left(a^{i}, g\right) \begin{cases}=0 & \text { for } a^{i} \leq \tilde{a}^{*}(g) ; \\ >0 & \text { for } a^{i}>\tilde{a}^{*}(g),\end{cases}
$$

where $e_{a}^{*}\left(a^{i}, g\right)>0$ and $e_{g}^{*}\left(a^{i}, g\right)>0$ in the second case.

Now considering the case of constrained optimization, the first-order condition for $e^{i}$ is, as follows from Eqs. 13 and 14,

$$
G\left(e^{i}, a^{i}, g\right) \equiv G^{*}\left(e^{i}, a^{i}, g\right)+\frac{e^{p}-e^{i}}{1-\alpha} h_{e}\left(e^{i}, a^{i}, g\right) \leq 0,
$$

with equality if $e^{i}>0$. Now that the ex ante education decision $e^{p}=e^{*}(\bar{a}, g)$ is continuous and increases with respect to $g$, the ex post education decision $e^{i}$ is written as a continuous function $e\left(a^{i}, g\right)$, which shares the qualitative properties with $e^{*}\left(a^{i}, g\right)$ derived above. ${ }^{36}$

By using $e^{*}\left(a^{i}, g\right)$ and $e\left(a^{i}, g\right)$ above, Fig. 5 represents how the irreversibility constraint affects the relationship of education with ability and technology growth. The solid and the broken lines respectively indicate the cases with and without the constraint. First, consider the economy with a low growth rate of technology, $g^{l}$. Education investment is limited to some households who draw ability levels above $\bar{a}$. Since $e^{i} \leq e^{*}\left(a^{i}, g\right)$ for all $a^{i} \in[0,1]$ with strict inequality for $a^{i}>\tilde{a}^{*}(g)$, the constraint encourages under-investment in education. This situation therefore corresponds to stage I in Fig. 3.

Next, consider a higher growth rate of technology $g^{h}$. Education investment is now widespread and is attractive even for some households observing $a^{i}<\bar{a}$. In the sense that $e^{i} \geq e^{*}\left(a^{i}, g\right)$ for all $a^{i} \in[0, \bar{a})$ with strict inequality for some $a^{i}$, the situation is similar to stage II in Fig. 4. In contrast, unlike in Fig. 4, the constraint prevents education investment by households of above-average children; i.e., $e^{i}<e^{*}\left(a^{i}, g\right)$ for all $a^{i}>\bar{a}$. After all, the economy faces not only over-investment but also underinvestment in education. However, the latter would be less significant for aggregate human capital unless the ability distribution is left skewed, noting the decreasing marginal productivity of education.

\footnotetext{
${ }^{35}$ Under the conditions on $h\left(e^{i}, a^{i}, g\right)$, there is a value $g>0$ such that $G^{*}\left(0, a^{i}, g\right)=0$ for any $a^{i} \in$ $(0,1)$. Hence the Implicit Function Theorem reveals the existence of a continuous function $\tilde{a}=\tilde{a}^{*}(g)$ such that $d \tilde{a}^{*}(g) / d g=-G_{g}^{*}\left(0, \tilde{a}^{*}, g\right) / G_{a}^{*}\left(0, \tilde{a}^{*}, g\right)<0$.

${ }^{36}$ As in Section 2.3.2, the ex ante education decision is made with the belief that children will be born with average ability $\bar{a}$.
} 


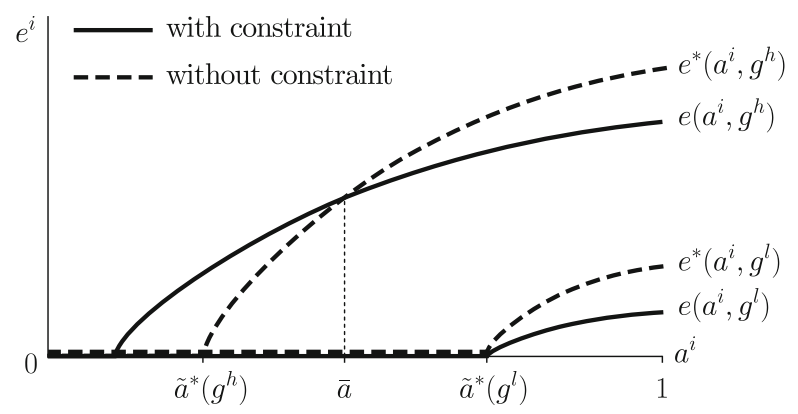

Fig. 5 The lock-in effects on education: a continuous case. The diagram illustrates how the lock-in effects on education change depending on the growth rate of technology $g$, when the education level increases continuously with ability $a^{i}$. When $g$ is at a relatively low level $g^{l}$, the irreversibility constraint has nonpositive impacts on individuals' education decisions as in Fig. 3. With a higher growth rate $g^{h}$, the lock-in effects are nonnegative for low-ability children $\left(a^{i}<\bar{a}\right)$ as in Fig. 4, although they are positive for high-ability children $\left(a^{i}>\bar{a}\right)$

\subsection{Heterogeneity of children}

The final part of the extensions incorporates heterogeneity among siblings and allows parents to make education decisions for each of them. In reality, such educational inequality between siblings would be conceivable at tertiary rather than primary levels.

For simplicity, suppose that adult individuals are identical in all aspects and each of them gives rise to a continuum of children whose ability levels are distributed according to the function $F$. In such circumstances, education is provided only for children above a certain ability level, implying that education investment is no longer the "all or nothing" choice for each household. Despite the fine tuning of education expenditures, however, there remain both under- and over-investments in education in the economy.

\subsubsection{Unconstrained optimization (benchmark case)}

In the absence of the irreversibility constraint, each individual essentially makes a simultaneous decision on the quantity of children, $n$, and the critical ability level for education, $\tilde{a} \cdot{ }^{37}$ That is,

$$
\begin{aligned}
\{n, \tilde{a}\}= & \arg \max (1-\alpha) \ln \left\{h-\left[\delta+\int_{\tilde{a}}^{1} \bar{e} d F(a)\right] n\right\}+\alpha \ln n \\
& +\alpha \ln \left\{\int_{0}^{\tilde{a}}(\bar{h}-g) d F(a)+\int_{\tilde{a}}^{1} \bar{h}-(1-a) g d F(a)\right\} .
\end{aligned}
$$

The first-order condition for $n$ yields

$$
n=\frac{\alpha h}{\delta+\bar{e}[1-F(\tilde{a})]} .
$$

\footnotetext{
${ }^{37}$ Now that all adult individuals are identical, the superscript $i$ denoting individual $i$ is omitted in this subsection.
} 
Substituting the fertility decision into the objective function above, the optimization problem is reduced to

$$
\tilde{a}=\arg \max \frac{\bar{h}-g+\int_{\tilde{a}}^{1} \operatorname{agd} F(a)}{\delta+\bar{e}[1-F(\tilde{a})]},
$$

It follows that the first-order condition for $\tilde{a}$ is

$$
\begin{aligned}
G^{*}(\tilde{a}, g) & \equiv-\tilde{a} g\{\delta+\bar{e}[1-F(\tilde{a})]\}+\bar{e}\left[\bar{h}-g+\int_{\tilde{a}}^{1} \operatorname{agdF}(a)\right] \\
& =0
\end{aligned}
$$

Noting that $G^{*}(1, \bar{e} \bar{h} /(\delta+\bar{e}))=0$, one finds that $G^{*}(0, g)>0, G^{*}(1, g)<0$, $G_{\tilde{a}}^{*}(\tilde{a}, g)<0$ and $G_{g}^{*}(\tilde{a}, g)<0$ for any $\tilde{a} \in(0,1)$ and $g \in(\bar{e} \bar{h} /(\delta+\bar{e}), \bar{h}]$. Hence, according to the Implicit Function Theorem, the optimal $\tilde{a}$ is given by a single-valued function $\tilde{a}^{*}(g)$ such that $\tilde{a}^{*}(\bar{e} \bar{h} /(\delta+\bar{e}))=1, \tilde{a}^{*}(\bar{h})>0$, and $d \tilde{a}^{*}(g) / d g<0$ for any $g \in(\bar{e} \bar{h} /(\delta+\bar{e}), \bar{h}]$.

\subsubsection{Constrained optimization: education investment}

In the presence of the irreversibility constraint, the fertility decision is made as in the mainline model in Section 2.3.2. For this reason, the analysis here focuses on the ex post education decision. The optimization problem is, for given $n$ and $g$,

$$
\begin{aligned}
\tilde{a}= & \arg \max (1-\alpha) \ln \left\{h-\left[\delta+\int_{\tilde{a}}^{1} \bar{e} d F(a)\right] n\right\} \\
& +\alpha \ln \left\{\int_{0}^{\tilde{a}}(\bar{h}-g) d F(a)+\int_{\tilde{a}}^{1} \bar{h}-(1-a) g d F(a)\right\} .
\end{aligned}
$$

If fertility decisions are made with no education planning as in Section 3.1, $n=\alpha h / \delta$ and the first-order condition is expressed as

$$
G^{I}(\tilde{a}, g)=G^{*}(\tilde{a}, g)+\frac{\bar{e} \tilde{a} g}{1-\alpha}[1-F(\tilde{a})]=0,
$$

where $F(1)=1$. This implies that $\tilde{a}$ is expressed as a function $\tilde{a}^{I}(g)$ such that $\tilde{a}^{I}(\bar{e} \bar{h} /(\delta+\bar{e}))=1, d \tilde{a}^{I}(g) / d g<0$ and $\tilde{a}^{I}(g)>\tilde{a}^{*}(g)$ for any $g \in(\bar{e} \bar{h} /(\delta+\bar{e}), \tilde{g}]$, where $\tilde{g} \equiv \bar{e} \bar{h} /(\bar{a} \delta+\bar{e})$ from Eq. 12 . In contrast, if the smaller number of children, $n=\alpha h /(\delta+\bar{e})$, is chosen as in Section 3.2, the first-order condition is

$$
G^{I I}(\tilde{a}, g)=G^{*}(\tilde{a}, g)-\frac{\bar{e} \tilde{a} g}{1-\alpha} F(\tilde{a})=0,
$$

where $F(0)=0$. This condition implies that $\tilde{a}$ is expressed as a function $\tilde{a}^{I I}(g)$ such that $d \tilde{a}^{I I}(g) / d g<0$ and $0<\tilde{a}^{I I}(g)<\tilde{a}^{*}(g)$ for any $g \in(\bar{e} \bar{h} /(\delta+\bar{e}), \bar{h}]$. 
Those results show that $\tilde{a}^{*}(g), \tilde{a}^{I}(g)$ and $\tilde{a}^{I I}(g)$ essentially have the same relationships as those depicted by Fig. 2. As long as the fertility decision is irreversible, under- or over-investment in education, respectively, occurs depending on whether $g$ is greater than $\tilde{g}$ or not. ${ }^{38}$

\section{Concluding remarks}

From the long-run perspective, this theoretical research has elucidated the role of irreversible fertility decisions in the formation of aggregate human capital, which comprises the quantity and quality of labor. With unexpected ability shocks on children, the irreversibility constraint affects parental decisions on child rearing and distorts the resource allocation between children's quantity and quality. The nature of the inefficiency varies with the stage of economic development. Depending on the growth rate of technology, the economy undergoes either over- or under-investment in education. The resource allocation can be improved by redistributional policies that alter the cost of child rearing.

While the central thesis of the present research is intuitive, the theory developed above builds on several simplifying assumptions. The first assumption is that individuals have no retirement period. If they lived on public pensions after retirement, the pension benefits they receive would depend on the proportion of the senior to the working population. This would be another reason for under-investment in the quantity of children to be reformed. The second is that the economy is not exposed to any demographic changes attributable to, for instance, immigration or emigration. The former would increase the working population, whereas the latter would be associated with a brain drain. It is worth investigating how they affect the macroeconomic problem of resource allocation between the quantity and quality of labor. The third is that the theory abstracts from endogenous growth. The irreversibility constraint on fertility decisions would intervene dynamically between population, education, and economic growth on the ground that aggregate human capital is presumably one of the prime factors of technological progress. These issues should be addressed in future research.

\section{Appendix 1: Proofs of the key results}

Proof of Lemma 2 Consider an adult individual $i$ who does not face the irreversibility constraint. Equation 6 implies that given $g>0$ and $e^{i}=\bar{e}$, the individual expects to obtain more utility by choosing $n^{i}=\frac{\alpha}{\delta+\bar{e}} h^{i}$ than by choosing $n^{i}=\frac{\alpha}{\delta} h^{i}$, which is a feasible choice under (A1). Applying this result to Eq. 5 reveals that $\kappa^{I}>\bar{e} / \delta$. Then, the lemma follows from Eqs. 9 and 21.

\footnotetext{
${ }^{38}$ If $g$ is smaller than $\bar{e} \bar{h} /(\delta+\bar{e})$, the irreversibility constraint is unbinding: In this case, $\tilde{a}^{I}(g)>\tilde{a}^{*}(g)>1$ and thus no one invests in education regardless of the constraint.
} 
Proof of Lemma 3 Applying the implicit function theorem to Eq. 25 yields

$$
\partial \tilde{a}^{I}(g ; 0) / \partial \delta^{g}=-\frac{(1-\alpha)\left(1+\kappa^{I}\right)}{\delta-\alpha(\delta+\bar{e})}\left[\frac{\bar{e}}{\delta}+\frac{1}{1-F\left(\tilde{a}^{I}(g)\right)}\right] \frac{\bar{h}-g}{g} .
$$

where $\tilde{a}^{I}(g)$ is given by Eq. 21 . The result is obtained by noting that $\tilde{a}^{I}(g)<1$ and $F\left(\tilde{a}^{I}(g)\right)<1 \forall g>\hat{g}$.

Proof of Lemma 4 Consider an adult individual $i$ who does not face the irreversibility constraint. Equation 6 implies that given $g>0$ and $e^{i}=0$, the individual expects to obtain more utility by choosing $n^{i}=\frac{\alpha}{\delta} h^{i}$ than by choosing $n^{i}=\frac{\alpha}{\delta+\bar{e}} h^{i}$. Applying this result to Eq. 5 reveals that $\kappa^{I I}<\bar{e} / \delta$. Then, the lemma follows from Eqs. 9 and 28.

Proof of Lemma 5 Applying the implicit function theorem to Eq. 30 yields

$$
\partial \tilde{a}^{I I}(g ; 0) / \partial \delta^{g}=-\frac{1+\kappa^{I I}}{\delta+\bar{e}}\left[\frac{(1-\alpha) \bar{e}}{\delta+\bar{e}-\alpha \delta}+\frac{1}{F(\bar{a})-F\left(\tilde{a}^{I I}(g)\right)}\right] \frac{\bar{h}-g}{g},
$$

where $\tilde{a}^{I I}(g)$ is given by Eq. 28 . The result is obtained respectively by noting that $\tilde{a}^{I I}(g)<\bar{a}$ and $F\left(\tilde{a}^{I I}(g)\right)<F(\bar{a}) \forall g>\tilde{g}$.

\section{Appendix 2: The model with expected utility}

Equation 33 in Section 4.1 is derived by dividing the ex ante optimization problem into several cases, depending on whether $\tilde{a}\left(n^{i}, g\right)$ in Eq. 15 is greater than unity. In order to facilitate the analysis below, let $\hat{n}^{i}$ be the critical value of $n^{i}$ such that $\tilde{a}\left(n^{i}, g\right)=1$. Using Eq. 15, one finds that

$$
\hat{n}^{i}=\frac{(1-\lambda) h^{i}}{(1-\lambda) \delta+\bar{e}}, \quad \text { where } \lambda \equiv\left(\frac{\bar{h}-g}{\bar{h}}\right)^{\frac{\alpha}{1-\alpha}} .
$$

Note that $0<\hat{n}^{i}<h^{i} /(\delta+\bar{e})$ and $\partial \hat{n}^{i} / \partial g>0 \forall g \in(0, \bar{h})$. Moreover, $\hat{n}^{i}=\alpha h^{i} / \delta$ if $g=\hat{g}$ because, given Eqs. 21 and $15, \hat{g}$ is a critical value on $(0, \bar{h})$ such that $\tilde{a}^{I}(\hat{g})=\tilde{a}\left(\alpha h^{i} / \delta, \hat{g}\right)=1$.

\section{Case 1: $g \in(0, \hat{g}]$}

Case 1-1 considers the optimal fertility choice in the interval $\left[\hat{n}^{i}, \infty\right)$. Since $\tilde{a}\left(n^{i}\right.$, $g$ ) $\geq 1$ in Eq. 32, the first-order condition is simplified to

$$
D\left(n^{i}\right) \equiv \frac{\alpha}{1-\alpha} \frac{1}{n^{i}}-\frac{\delta}{h^{i}-n^{i} \delta}=0,
$$

where $D\left(n^{i}\right)$ is a strictly decreasing function such that $D\left(\hat{n}^{i}\right) \geq 0$ with equality if and only if $g=\hat{g}$ (and thus $\hat{n}^{i}=\alpha h^{i} / \delta$ ). Thus, the optimal choice in this interval is $n^{i}=\alpha h^{i} / \delta \geq \hat{n}^{i}$, with equality if and only if $g=\hat{g}$. 
Case 1-2 considers the optimal fertility choice in $\left(0, \hat{n}^{i}\right]$. Noting that $0<$ $\tilde{a}\left(n^{i}, g\right) \leq 1$ under the circumstances, differentiating the objective function in Eq. 32 with respect to $n^{i}$ and arranging the result yield $G\left(\hat{n}^{i}, g\right) \geq 0$, with equality if and only if $g=\hat{g}$, where

$$
\begin{aligned}
G\left(n^{i}, g\right) \equiv & \frac{\alpha}{1-\alpha} \frac{1}{n^{i}}-\tilde{a}\left(n^{i}, g\right) \frac{\delta}{h^{i}-n^{i} \delta} \\
& -\left[1-\tilde{a}\left(n^{i}, g\right)\right] \frac{\delta+\bar{e}}{h^{i}-n^{i}(\delta+\bar{e})} .
\end{aligned}
$$

In the following, the second-order condition is imposed to ensure the uniqueness of the solution. That is, for any $\left(n^{i}, g\right)$ such that $0<\tilde{a}\left(n^{i}, g\right) \leq 1$ and $G\left(n^{i}, g\right)=0$,

$$
\begin{aligned}
G_{n}\left(n^{i}, g\right) & =-\frac{\alpha}{1-\alpha} \frac{1}{\left(n^{i}\right)^{2}} \\
& +\tilde{a}_{n}\left(n^{i}, g\right) \frac{\bar{e} h^{i}}{\left(h^{i}-n^{i} \delta\right)\left[h^{i}-n^{i}(\delta+\bar{e})\right]} \\
& -\tilde{a}^{i} \frac{\delta^{2}}{\left(h^{i}-n^{i} \delta\right)^{2}}-\left(1-\tilde{a}^{i}\right) \frac{(\delta+\bar{e})^{2}}{\left[h^{i}-n^{i}(\delta+\bar{e})\right]^{2}} \\
& <0 .
\end{aligned}
$$

Then, $G\left(n^{i}, g\right)>0 \forall n^{i} \in\left(0, \hat{n}^{i}\right)$ and thus $n^{i}=\hat{n}^{i}$ is the optimal choice in this interval. ${ }^{39}$

Given the two results above, consider the fertility choice for the entire interval $(0, \infty)$. Since the objective function in Eq. 32 is continuous at $\hat{n}^{i}$, one finds that $n^{i}=\alpha h^{i} / \delta$ is globally optimal for $g \in(0, \hat{g}]$.

\section{Case $2: g \in(\hat{g}, \bar{h})$}

Case 2-1 considers the optimal fertility choice in the interval $\left[\hat{n}^{i}, \infty\right)$. Since $\tilde{a}\left(n^{i}, g\right) \geq 1$, differentiating the objective function in Eq. 32 with respect to $n^{i}$ and arranging the result reveals that $D\left(n^{i}\right)<0$ in this interval. Hence, the optimal choice is $n^{i}=\hat{n}^{i}$.

Case 2-2 considers the optimal fertility choice on $\left(0, \hat{n}^{i}\right]$. Since $0<\tilde{a}\left(n^{i}, g\right) \leq 1$, the optimality condition is $G\left(n^{i}, g\right)=0$, where $G(\cdot)$ is a continuous function such that $G\left(\hat{n}^{i}, g\right)<0$ and $G\left(n^{i}, g\right) \rightarrow \infty$ as $n^{i} \rightarrow 0$. This first-order condition is therefore satisfied by a value of $n^{i}$ on $\left(0, \hat{n}^{i}\right)$. One may find the value by guessing that $n^{i}$ is proportional to $h^{i}$; i.e., $n^{i}=\gamma h^{i}$. Then, the first-order condition is simplified to

$$
\frac{\alpha}{1-\alpha} \frac{1}{\gamma}-\tilde{a}^{i} \frac{\delta}{1-\gamma \delta}-\left(1-\tilde{a}^{i}\right) \frac{\delta+\bar{e}}{1-\gamma(\delta+\bar{e})}=0,
$$

where $\tilde{a}^{i}=\tilde{a}\left(\gamma h^{i}, g\right)$ is independent of $h^{i}$ (cf. Eq. 15). Given the second-order condition in Eq. 36, along with the property that $\tilde{a}_{g}\left(n^{i}, g\right)<0$, the first-order condition

\footnotetext{
${ }^{39}$ The second-order condition in Eq. 36 is satisfied when $\bar{e}$ is sufficiently small. Such a restriction on $\bar{e}$ is compatible with the other key assumptions (cf. Footnote 24).
} 
implies a one-to-one negative relationship of $\gamma$ to $g \in[\hat{g}, \bar{h}]$. In particular, note that $\gamma=\alpha / \delta$ if $g=\hat{g}$ and that $\gamma=\alpha /(\delta+\bar{e})$ if $g=\bar{h} .{ }^{40}$

With the two results above, consider the fertility choice for the entire interval $(0, \infty)$. Because the objective function is continuous at $\hat{n}^{i}, n^{i}=\gamma h^{i}<\alpha h^{i} / \delta$ is globally optimal for $g \in(\hat{g}, \bar{h})$.

Acknowledgements The authors are grateful to Alessandro Cigno (the editor), two anonymous referees, Junko Doi, Shin-ichi Fukuda, Akira Momota, Atsuhiro Taki, Robert Tamura, Yasunobu Tomoda, Ng Yew Kwang, and seminar participants at Hitotsubashi University, Kobe University, Kansai University, the University of Tokyo, the 13th Macroeconomics Conference, the 2016 Spring Meeting of Japan Association for Applied Economics, 2016 Taipei International Conference on Growth, Trade and Dynamics, the 2016 Asian Meeting of the Econometric Society, Hiroshima-NTU Joint Economics Workshop, the 18th annual congress of the Association for Public Economic Theory, and the Singapore Economic Review Conference 2017, for their valuable comments and encouragement.

Funding This work was supported by JSPS KAKENHI Grant Number JP19K01567 and by the Kansai University Fund for Domestic and Overseas Research Fund, 2020.

\section{Declarations}

Conflict of interest The authors declare no competing interests.

\section{References}

Aiyagari SR, Greenwood J, Seshadri A (2002) Efficient investment in children. J Econ Theory 102(2):290-321. https://doi.org/10.1006/jeth.2001.2852

Ashraf QH, Weil DN, Wilde J (2013) The effect of fertility reduction on economic growth. Popul Dev Rev 39(1):97-130. https://doi.org/10.1111/j.1728-4457.2013.00575.x

Becker GS, Lewis HG (1973) On the interaction between the quantity and quality of children. J Polit Econ 81(2.2):S279-S288. https://doi.org/10.1086/260166

Chevalier A (2003) Measuring over-education. Economica 70(279):509-531. https://doi.org/10.1111/ 1468-0335.t01-1-00296

Cigno A, Luporini A (2019) Student loans and the allocation of graduate jobs. Can J Econ 52(1):339-378. https://doi.org/10.1111/caje.12374

Clark G (2012) Japan's university education crisis. The Japan Times 15

Dảvila J (2018) Internalizing fertility and education externalities on capital returns. Econ Theory 66(2):343-373. https://doi.org/10.1007/s00199-017-1062-z

de la Croix D, Doepke M (2009) To segregate or to integrate: Education politics and democracy. Rev Econ Stud 76(2):597-628. https://doi.org/10.1111/j.1467-937X.2008.00529.x

Doepke M (2005) Child mortality and fertility decline: Does the Barro-Becker model fit the facts? J Popul Econ 18(2):337-366. https://doi.org/10.1007/s00148-004-0208-Z

Doepke M, Zilibotti F (2005) The macroeconomics of child labor regulation. Am Econ Rev 95(5):14921524. https://doi.org/10.1257/000282805775014425

Fraser CD (2001) Income risk, the tax-benefit system and the demand for children. Economica 68(269):105-125. https://doi.org/10.1111/1468-0335.00236

Galor O, Moav O (2000) Ability-biased technological transition, wage inequality, and economic growth. Q J Econ 115(2):469-497. https://doi.org/10.1162/003355300554827

Galor O, Weil DN (2000) Population, technology, and growth: from Malthusian stagnation to the demographic transition and beyond. Am Econ Rev 90(4):806-828. https://doi.org/10.1257/aer.90.4.806

\footnotetext{
${ }^{40}$ The first and second properties are, respectively, obtained by noting that $\tilde{a}\left(\alpha h^{i} / \delta, \hat{g}\right)=1$ and that $\tilde{a}\left(\gamma h^{i}, \bar{h}\right)=0 \forall \gamma \in(0,1 /(\delta+\bar{e}))$.
} 
Galor O, Zeira J (1993) Income distribution and macroeconomics. Rev Econ Stud 60(1):35-52. https://doi.org/10.2307/2297811

Goldstein J, Lutz W, Testa MR (2003) The emergence of sub-replacement family size ideals in Europe. Popul Res Policy Rev 22(5/6):479-496. https://doi.org/10.1023/B:POPU.0000020962.80895.4a

Gould ED, Moav O, Weinberg BA (2001) Precautionary demand for education, inequality, and technological progress. J Econ Growth 6(4):285-315. https://doi.org/10.1023/A:1012782212348

Hagewen KJ, Morgan SP (2005) Intended and ideal family size in the United States, 1970-2002. Popul Dev Rev 31(3):507-527. https://doi.org/10.1111/j.1728-4457.2005.00081.x

Hopkins E (2012) Job market signaling of relative position, or Becker married to Spence. J Eur Econ Assoc 10(2):290-322. https://doi.org/10.1111/j.1542-4774.2010.01047.x

Iyigun MF (2000) Timing of childbearing and economic growth. J Dev Econ 61(1):255-269. https://doi.org/10.1016/S0304-3878(99)00066-8

Joshi S, Schultz TP (2013) Family planning and women's and children's health: long-term consequences of an outreach program in Matlab, Bangladesh. Demography 50(1):149-180. https://doi.org/10.1007/ s13524-012-0172-2

Kalemli-Ozcan S (2003) A stochastic model of mortality, fertility, and human capital investment. J Dev Econ 70(1):103-118. https://doi.org/10.1016/S0304-3878(02)00089-5

Moav O (2002) Income distribution and macroeconomics: The persistence of inequality in a convex technology framework. Econ Lett 75(2):187-192. https://doi.org/10.1016/S0165-1765(01)00625-5

Moav O (2005) Cheap children and the persistence of poverty. Econ J 115(500):88-110. https://doi.org/10. 1111/j.1468-0297.2004.00961.x

Montenegro CE, Patrinos HA (2014) Comparable estimates of returns to schooling around the world. World Bank Policy Research Working Paper 7020. https://doi.org/10.1596/1813-9450-7020

Mookherjee D, Ray D (2003) Persistent inequality. Rev Econ Stud 70(2):369-393. https://doi.org/10.1111/ 1467-937X.00248

Nakagawa M, Sugimoto Y (2011) Income distribution and macroeconomics: Fertility adjustment prior to education investment. Econ Lett 110(2):155-158. https://doi.org/10.1016/j.econlet.2010.11.014

National Institute of Population and Social Security Research (2017) Marriage and childbirth in Japan today : the fifteenth Japanese national fertility survey, 2015 (results of singles and married couples survey). Tech. rep., National Institute of Population and Social Security Research, Tokyo, http://www. ipss.go.jp/ps-doukou/j/doukou15/NFS15_reportALL.pdf

Nelson RR, Phelps ES (1966) Investment in humans, technological diffusion, and economic growth. Am Econ Rev 56(1/2):69-75. http://www.jstor.org/stable/1821269

Promotion and Mutual Aid Corporation for Private Schools of Japan (2019) Heisei 31 (2019) nendo shiritsu daigaku tanki daigaku to nyugaku shigan doko (trends in applicants for admission to private universities and junior colleges in 2019). Tech. rep.. https://www.shigaku.go.jp/files/shigandoukouH31. pdf

Sicherman N (1991) Overeducation in the labor market. J Labor Econ 9(2):101-122. https://doi.org/10. $1086 / 298261$

United Nations (2016) The millennium development goals report 2015. United Nations https://doi.org/10. $18356 / 6 \mathrm{~cd} 11401-\mathrm{en}$

United Nations (2017) World population prospects: the 2017 revision, volume I: comprehensive tables (ST/ESA/SER.A/399). United Nations, New York, https://esa.un.org/unpd/wpp/Publications/Files/ WPP2017_Volume-I_Comprehensive-Tables.pdf

Publisher's note Springer Nature remains neutral with regard to jurisdictional claims in published maps and institutional affiliations. 